\title{
Macroscopic description of particle systems with nonlocal density-dependent diffusivity
}

\author{
Cristóbal López \\ Instituto Mediterráneo de Estudios Avanzados IMEDEA (CSIC-UIB), Campus de la Universidad de las Islas Baleares, \\ E-07122 Palma de Mallorca, Spain
}

(Received 21 March 2006; published 20 July 2006)

\begin{abstract}
In this paper we study macroscopic density equations in which the diffusion coefficient depends on a weighted spatial average of the density itself. We show that large differences (not present in the local densitydependence case) appear between the density equations that are derived from different representations of the Langevin equation describing a system of interacting Brownian particles. Linear stability analysis demonstrates that under some circumstances the density equation interpreted with the Ito calculus has pattern solutions that never appear for the kinetic interpretation, which is the other one typically appearing in the context of nonlinear diffusion processes. We also introduce a discrete-time microscopic model of particles that confirms the results obtained at the macroscopic density level.
\end{abstract}

DOI: 10.1103/PhysRevE.74.012102

PACS number(s): 05.40.-a, 87.23.Cc, 05.10.Gg

In the mathematical description of many ecological systems the mobility of the species depends on the local density or concentration of individuals [1,2]. A standard example of this is given by models for animal or insect dispersal where it is usually considered that there is an increase of the diffusion coefficient due to population pressure. These models are written down as an extension of the diffusion equation which includes a diffusivity depending on the population density. Under the generic name of nonlinear diffusion equations similar equations arise in other contexts, as in the flow through a porous medium, bacterial dynamics, and transport in plasmas [2,3].

Generally, the nonlinear diffusion equations for the density correspond to a coarse-grained description of the more fundamental particle stochastic dynamics. Similarly to the case of diffusion in nonhomogeneous media where the diffusion coefficient varies in space, a heuristic derivation of the nonlinear diffusion density equations has to deal with the Ito-Stratonovich dilemma $[4,5]$. (To be precise one should speak about the interpretation of the Langevin equation since, as we will see later, the Stratonovich realization does not appear in our study and one has instead the kinetic interpretation of the Langevin equation [6].) In the context of noise-induced phase transitions this is of major importance [7], and this work aims to show that it is also specially relevant when one considers nonlocal interactions between the particles.

In a biological setting, nonlocal effects have been widely considered since they account for the interaction between individuals that are separated in space [8]. More recently, there has appeared interest in models where the mobility depends on an averaged density rather than on its value at a point [9]. The spatial nonlocality is there introduced in order to take into account the finite size of the particles or the senses (visual, hearing, etc.) of the organisms, which somehow determine their mobility. The idea behind this is that the diffusivity of any particle may depend on the configuration of other particles in its vicinity.

Thus, in this work we study a nonlocal version of a general density-dependent diffusion equation for the number density of individuals. We focus on its pattern and clustering formation properties. Making a linear stability analysis we first show that, contrary to what happens in the local case, completely different results can be obtained for the Ito and kinetic descriptions. In fact, spatial patterns can emerge for the first but never for the second. Then, we introduce two microscopic interacting particle systems whose continuum evolutions are given by the Ito and kinetic density equations. Numerical simulations of these systems confirm the calculations made with the continuum density equations.

Let us consider a population dynamics model of relevance for insect dispersal. The diffusion coefficient depends on the local density of particles, i.e., $D=D(\rho)$, where $\rho(\mathbf{x}, t)$ is the population density (we assume that $D$ is not an explicit function of the spatiotemporal coordinates). We only study positive density dependence, which is the most widespread hypothesis and indicates that competition increases dispersal because individuals have better survival rates by leaving high-density areas [10]. Mathematically, $D^{\prime}(\rho) \equiv d D / d \rho>0$. The mean-field density descriptions in the Ito and kinetic interpretations are $[4,6,7]$

$$
\begin{gathered}
\frac{\partial \rho(\mathbf{x}, t)}{\partial t}=\nabla^{2}[D(\rho) \rho], \\
\frac{\partial \rho(\mathbf{x}, t)}{\partial t}=\nabla \cdot[D(\rho) \nabla \rho] .
\end{gathered}
$$

The first one corresponds to the Ito and the second to the kinetic interpretation, and in the following we will denote them, respectively, as the IE and KE. The KE is the one that is usually considered when modeling at the macroscopic level $[1,2]$, since one writes down the density evolution as a flux equation, $\partial_{t} \rho=-\nabla \cdot \mathbf{J}$, with the density-dependent flux $\mathbf{J}=-D(\rho) \boldsymbol{\nabla} \rho$. But this is not a definitive argument and, as discussed in [11], the IE should be the correct one for a continuum description of a discrete population dynamics model of nonoverlapping generations. A proper derivation of density equations from the fundamental stochastic particle dynamics, once a right interpretation for this is assumed, can be consulted in [12]. Note that they are stochastic, but in our study we neglect the fluctuations by considering a kind of mean-field approximation [13]. Let us assume an initial con- 
stant density $\rho(\mathbf{x}, 0)=\rho_{0}$. We now perform a linear stability analysis of the stationary homogeneous (identical to $\rho_{0}$ because particle number is conserved) solution for both the IE and KE. Writing $\rho(\mathbf{x}, t)=\rho_{0}+\epsilon \psi(\mathbf{x}, t)$, where $\epsilon$ is a small parameter and $\psi$ the space-time-dependent perturbation, we obtain (we label the equations with IE or KE)

$$
\begin{gathered}
(\mathrm{IE}): \quad \partial_{t} \psi(\mathbf{x}, t)=\left[D\left(\rho_{0}\right)+\rho_{0} D^{\prime}\left(\rho_{0}\right)\right] \nabla^{2} \psi, \\
(\mathrm{KE}): \quad \partial_{t} \psi(\mathbf{x}, t)=D\left(\rho_{0}\right) \nabla^{2} \psi .
\end{gathered}
$$

Note that in both the perturbation follows a simple diffusion equation and there is, therefore, no instability in the density equation that could give rise to the formation of patterns or aggregates of particles. From this point of view, the Ito and kinetic frameworks [Eqs. (1) and (2)] are equivalent.

Now let us consider that the diffusivity depends on an averaged density over the whole system, $\bar{\rho}(\mathbf{x}, t)$ $=\int_{\Re d} d \mathbf{r} G(\mathbf{x}-\mathbf{r}) \rho(\mathbf{r}, t)$, where the kernel $G(\mathbf{x}-\mathbf{r})$ represents the effect of population density at $\mathbf{r}$ on the density at $\mathbf{x}$. It embodies the forces of attraction and repulsion of neighbors [14]. We assume that $G$ is normalized so that $\int_{\Re^{d} d} d \mathbf{r} G(\mathbf{r})$ $=1$. Then let us consider $D=D(\bar{\rho})$ in Eqs. (1) and (2) and make again a linear stability analysis around $\rho_{0}$ for the now nonlocal KE and IE,

(KE)

$$
\boldsymbol{\epsilon} \partial_{t} \psi(\mathbf{x}, t)=\nabla\left[D\left(\rho_{0}+\epsilon \int d \mathbf{r} G(\mathbf{x}-\mathbf{r}) \psi(\mathbf{r}, t)\right) \boldsymbol{\nabla}\left(\rho_{0}+\epsilon \psi\right)\right],
$$

so that to first order in $\epsilon$ the perturbation simply diffuses, $\partial_{t} \psi=D\left(\rho_{0}\right) \nabla^{2} \psi$, and no instability happens in the KE. However, when we consider the Ito description we have

(IE)

$$
\begin{aligned}
\epsilon \partial_{t} \psi(\mathbf{x}, t) & \\
\quad= & \nabla^{2}\left[D\left(\rho_{0}+\epsilon \int d \mathbf{r} G(\mathbf{x}-\mathbf{r}) \psi(\mathbf{r}, t)\right)\left[\rho_{0}+\epsilon \psi(\mathbf{x}, t)\right]\right] \\
& =\epsilon \nabla^{2}\left(D\left(\rho_{0}\right) \psi+\rho_{0} D\left(\rho_{0}\right) \int d \mathbf{r} G(\mathbf{x}-\mathbf{r}) \psi(\mathbf{r}, t)\right) .
\end{aligned}
$$

Considering a harmonic perturbation $\psi(\mathbf{x}, t)=\exp (\lambda t+i \mathbf{k} \cdot \mathbf{x})$ it is not difficult to obtain the following dispersion relation:

$$
\lambda(k)=-k^{2}\left[D\left(\rho_{0}\right)+\rho_{0} D^{\prime}\left(\rho_{0}\right) \hat{G}(\mathbf{k})\right],
$$

where $k=|\mathbf{k}|$ and $\hat{G}(\mathbf{k})=\int d \mathbf{r} G(\mathbf{r}) e^{-i \mathbf{k} \cdot \mathbf{r}}$ is the Fourier transform of the kernel. The important fact with Eq. (7) is that depending on the kernel function [note that the form of the diffusivity functional is almost irrelevant once we have assumed that it is an increasing function of the density, $D^{\prime}(\rho)>0$ ], the perturbation growth rate can be positive, giving rise to the aggregation of particles or formation of spatial patterns $[8,14]$. As we have just seen, this is absolutely different from the result obtained in the kinetic framework Eq. (5) and below.

Next we introduce a model, choosing the density and the
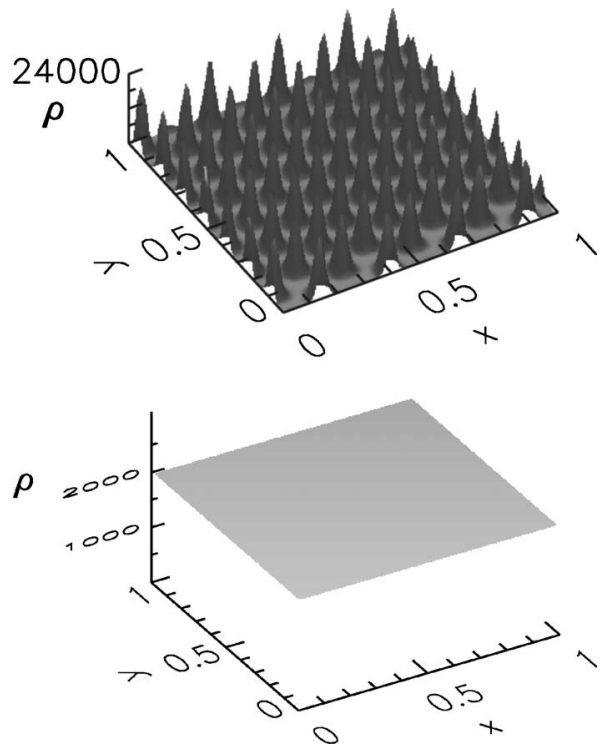

FIG. 1. Steady number density of particles with nonlocal density-dependence diffusivity. Top is the Ito and bottom the kinetic framework. The diffusivity functional is $D(\rho)=D_{0}\left(\rho / \rho_{0}\right)^{p}$, and we use the top-hat kernel with $R=0.1$. The values of the other parameters, in both plots, are $D_{0}=0.0001, p=9, \rho_{0}=2000$, and the system sizes in $x$ and $y$ are equal to 1 .

kernel functions, with a clearly oriented ecological application and where $\lambda$ can take positive values. We study the distribution of organisms over a spatial area and thus we restrict ourselves to two spatial dimensions. A typical form for $D(\rho)$ in animal dispersal models is $D_{0}\left(\rho / \rho_{0}\right)^{p}$ where $D_{0}$ and $p$ are positive real numbers [1,2], and $\rho_{0}$ is a reference density which we take equal to the initial one without loss of generality. Because of the normalization of the kernel function the averaged density $\bar{\rho}$ has the same dimensions as $\rho$. Concerning the kernel, we take the usual top-hat function [14] $G(\mathbf{r})=1 /\left(\pi R^{2}\right)$ if $|\mathbf{r}| \leqslant R$ and $G(\mathbf{r})=0$ otherwise, which introduces a typical interaction length $R$ in the system. One obtains $\hat{G}(k)=2 J_{1}(k R) /(k R)$ so that the dispersion relation takes the form

$$
\lambda(k)=-D_{0} k^{2}\left(1+\frac{2 p J_{1}(k R)}{k R}\right) .
$$

$J_{1}$ is the first-order Bessel function, and it is clear from Eq. (8) that the value of $D_{0}$ only sets the time scale of the system. The onset of pattern formation is $\lambda>0$ which numerically is approached for $p$ larger than $p_{c} \approx 7.6$. In Fig. 1 we show a long-time density solution for the IE (left) and KE (right) with this kernel and $D$ functions, and $p=9$. Note the hexagonal pattern formed in the IE and the homogeneous solution for the KE, i.e., the numerical results confirm the different spatial structures obtained for the two descriptions. We have checked that this is so for all $p$, as can be seen below in Fig. 3.

Up to now we have shown that, for nonlinear nonlocal diffusion phenomena, the different interpretations, in terms of density equations, of the same system dynamics lead to very distinct results. One may ask if this also happens at the 
level of the discrete interacting particle dynamics. Since the IE and KE are the mean-field density descriptions derived from the Langevin equation of a system of Brownian interacting particles, we expect that the same differences encountered before between both descriptions appear. We will show that this is in fact the case for two time-discrete microscopic systems which, in the continuous-time limit, are the Ito and kinetic Langevin equations corresponding to the IE and $\mathrm{KE}$ with the top-hat kernel and the typical diffusivity functional for insect dispersal.

Therefore, the problem is to find a discrete dynamics from which to derive the KE and IE. Following the discussion in [11] (Sec. 5.4.2) we first take a population dynamics system of nonoverlapping generations whose Langevin equation, in the time-continuum limit, should be interpreted in the Ito framework. The system consists of $N$ particles with positions $\mathbf{r}_{i}(t)=\left(x_{i}(t), y_{i}(t)\right), i=1, \ldots, N$, in a two-dimensional periodic box of size $L=1$ evolving as follows:

$$
\mathbf{r}_{i}(t+\Delta t)=\mathbf{r}_{i}(t)+\sqrt{2 D_{0}\left[N_{R}\left(\mathbf{r}_{i}(t)\right) / N\right]^{p} \Delta t} \boldsymbol{\eta}^{(i)}(t),
$$

where $D_{0}$ and $p$ are real positive numbers, $\Delta t$ is the time step, $N_{R}\left(\mathbf{r}_{i}(t)\right)$ is the number of particles at a distance less than $R$ of particle $i$ at time $t$, and $\boldsymbol{\eta}^{(i)}(t)=\left(\eta_{x}^{(i)}, \eta_{y}^{(i)}\right)$ is a Gaussian white noise with correlations $\left\langle\eta_{a}^{(i)}(t) \eta_{b}^{(j)}(s)\right\rangle$ $=\delta_{i j} \delta_{a b} \delta_{t s}$. As already mentioned, in the limit $\Delta t \rightarrow 0$ one obtains an Ito Langevin equation,

$$
\frac{d \mathbf{r}_{i}}{d t}=\sqrt{2 D_{0}\left[N_{R}\left(\mathbf{r}_{i}(t)\right) / N\right]^{p}} \boldsymbol{\eta}^{(i)}(t),
$$

and one can calculate $[12,13]$ that the corresponding density equation of this Langevin is the nonlocal IE with the top-hat kernel and the density functional of insect dispersal. For the kinetic case, the first step is to interpret Eq. (10) in this sense so that it is equivalent to the following Ito Langevin equation [15]:

$$
\frac{d \mathbf{r}_{i}}{d t}=g\left(\mathbf{r}_{i}(t)\right) \boldsymbol{\nabla}_{\mathbf{r}_{i}} g\left(\mathbf{r}_{i}(t)\right)+\sqrt{2 D_{0}\left[N_{R}\left(\mathbf{r}_{i}(t)\right) / N\right]^{p}} \boldsymbol{\eta}^{(i)}(t),
$$

where $g\left(\mathbf{r}_{i}(t)\right)=\sqrt{2 D_{0}\left[N_{R}\left(\mathbf{r}_{i}(t)\right) / N\right]^{p}}$.

Thus, our discrete-particle systems equivalent to the IE and KE are given by Eq. (9) and the discrete-time version up to order $\Delta t$ of Eq. (11), respectively. Note that, since our simulations are off lattice, i.e., particles move in the continuum space, we have to consider an auxiliary grid to compute the gradients of $g\left(\mathbf{r}_{i}(t)\right)$ for the kinetic case. In Fig. 2 we show the long-time spatial distribution of particles for the same parameter values used in Fig. 1. The particles following the time-discrete dynamics which give rise to the Ito Langevin equation are arranged in a hexagonal pattern of clusters, while in the kinetic case the particles are randomly distributed in space. This is completely in accord with what happens in the macroscopic description.

A more quantitative comparison between the macroscopic and microscopic dynamics follows. The characterization of the pattern can be performed via the structure function, whose maxima identify periodic structures. In the case of the
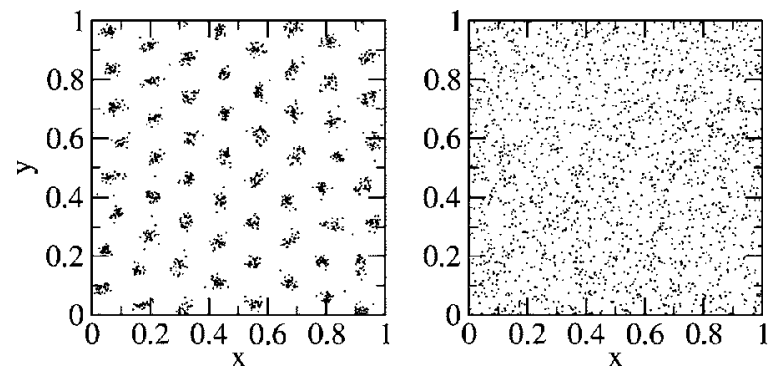

FIG. 2. Spatial distribution of particles at large time. Left is for Ito and right for kinetic description. We use the same parameters as in Fig. 1, i.e., $D_{0}=0.0001, p=9$, and $R=0.1$. The number of particles is $N=2000$ for both plots, and $d t=0.01$. For the right panel we take a square grid (to calculate the gradients of the $g$ function) with lattice spacing $d x=0.005$.

continuum density, the structure function in the steady state, $S_{c}$, is computed as the modulus of the Fourier transform of $\rho(\mathbf{x}, t)$ averaged spherically and in time. In the case of the particle system the structure function, which is related but not identical to $S_{c}$, is calculated as $S_{d}(k)=\left\langle\left|\sum_{j} e^{i \mathbf{k} \cdot \mathbf{x}_{j}(t)}\right|^{2} / N\right\rangle$, where $\mathbf{x}_{j}(t)$ is the position vector of particle $j, \mathbf{k}$ is a twodimensional wave vector with modulus $k$, and the average indicates a spherical average over the wave vectors with modulus $k$, a temporal average in the steady state, and an average over many different realizations. In Fig. 3 we plot the maximum (for $k>0$ ) value of $S_{c}$ (left panel) and of $S_{d}$ (right) versus the parameter $p$. With circles we plot the computed ones for the IE and with squares for the KE. It is clear from the plot that the value of $p$ at which the transition to pattern, in the Ito approach, occurs is the same for both, and coincident with the numerical value obtained from stability analysis. Moreover, for any value of $p$ no peak (i.e., no pattern) is ever observed in the kinetic prescription.

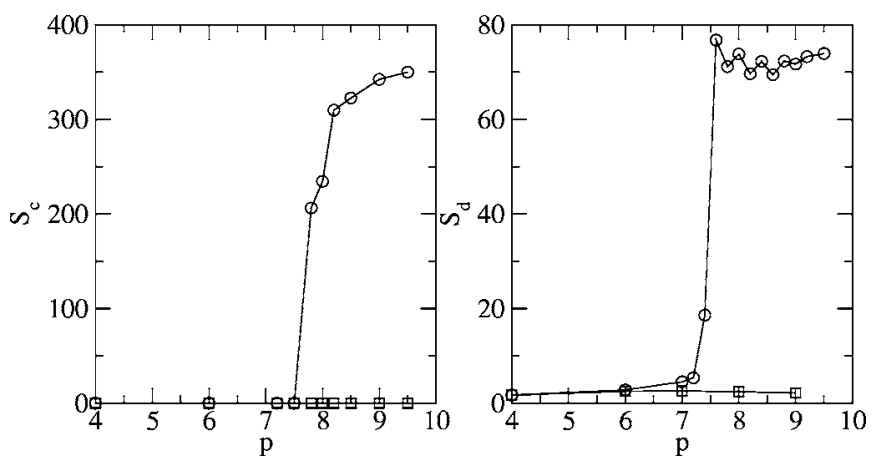

FIG. 3. Maxima of the structure functions for different values of the parameter $p$. In the left panel we plot the maximum value of $S_{c}$, i.e., the value corresponding to the continuum density equation. It is normalized with the number of points of the grid used to make the computation, 10 201. Circles are for the Ito prescription and squares for the kinetic one. On the right, we plot, also vs $p$, the maximum value of $S_{d}$, i.e., of the structure function for a distribution of particles. Again circles are for the Ito and squares for the kinetic approach. The other parameters for both plots are $D_{0}=0.0001$ and $R=0.1$. For the kinetic particle dynamics we use a lattice with $d x=0.005$. 
In summary, density evolution equations with densitydependent diffusion coefficient are standards in the macroscopic modeling of biological, physical, and chemical processes. Typically, they are postulated as $\partial_{t} \rho(\mathbf{x}, t)=-\boldsymbol{\nabla} \cdot \mathbf{J}$ with $\mathbf{J}$ the population flux given by $\mathbf{J}=-D(\rho) \boldsymbol{\nabla} \rho$. In this way one obtains a macroscopic density equation in the so-called kinetic framework, which is different from the Ito one, Eq. (1). Though this last expression rarely appears in the literature, this is no reason to exclude it [4], and one has to resort to the real microscopic dynamics to infer the proper density equation describing the system dynamics. In fact, a proper macroscopic equation for population dynamics models of nonoverlapping generations goes through the Ito framework [11]. In this work we have shown that, in the context of biological population dynamics but easily extended to others, no relevant differences, at the level of clustering or pattern formation, arise between the two equations when the functional $D(\rho)$ is local. However, when $D$ is nonlocal the differ- ences are rather important, and nonhomogeneous spatial structures can appear in the Ito but never in the kinetic rule. Furthermore, we have discussed the discrete dynamics of nonlocally interacting-particle systems from which the Ito and kinetic density equations are derived, confirming also at this level the large differences encountered in the macroscopic description. It is thus clear that the main message of this work is that it is specially relevant in the case of systems with nonlocal density-dependent diffusivity that the macroscopic description should be properly derived from the discrete particle model. The inclusion of reaction terms and further extensions of the model will be pursued in the future.

I acknowledge useful conversations with Emilio Hernández-García and Umberto Marini Bettolo Marconi. Financial support from MEC (Spain) and FEDER through project CONOCE2 (Grant No. FIS2004-00953) is greatly acknowledged.
[1] A. Okubo and S. Levin, Diffusion and Ecological Problems: Modern Perspectives (Springer-Verlag, New York, 2001).

[2] J. D. Murray, Mathematical Biology (Springer-Verlag, Berlin, 2002).

[3] D. G. Aronson, in Nonlinear Diffusion Problems, edited by A. Fasano and M. Primicerio, Lecture Notes in Mathematics Vol. 1224 (Springer-Verlag, Berlin, 1985).

[4] N. G. van Kampen, Stochastic Processes in Physics and Chemistry, 2nd ed. (North-Holland, Amsterdam, 1992); N. Van Kampen, J. Phys. Chem. Solids 49, 673 (1988); C. W. Gardiner, Handbook of Stochastic Methods, 2nd ed. (SpringerVerlag, Berlin, 1985).

[5] J. M. Sancho, M. San Miguel, and D. Dürr, J. Stat. Phys. 28, 291 (1982)

[6] P. Hänggi and H. Thomas, Phys. Rep. 88, 207 (1982); Y. L. Klimontovich, Physica A 163, 515 (1990).

[7] C. Van den Broeck, J. M. R. Parrondo, and R. Toral, Phys. Rev. Lett. 73, 3395 (1994); C. Van den Broeck, J. M. R. Parrondo, R. Toral, and R. Kawai, Phys. Rev. E 55, 4084 (1997).

[8] G. Flierl, D. Grunbaum, S. Levin, and D. Olson, J. Theor. Biol. 196, 397 (1999); A. Mogiler and L. Edelstein-Keshet, J. Math. Biol. 38, 534 (1999); F. Cecconi, M. Marsili, J. R. Banavar, and A. Maritan, Phys. Rev. Lett. 89, 088102 (2002); C. Topaz and A. Bertozzi, SIAM J. Appl. Math. 65, 152 (2004). Y. E. Maruvka and N. M. Shnerb, Phys. Rev. E 73, 011903 (2006).

[9] D. D. Holm and V. Putkaradze, Phys. Rev. Lett. 95, 226106 (2005); D. D. Holm and V. Putkaradze, e-print nlin.PS/ 0506020; C. López, Phys. Rev. E 72, 061109 (2005).

[10] E. Matthysen, Ecography 28, 403 (2005).

[11] W. Horsthemke and R. Lefever, Noise-Induced Transitions (Springer-Verlag, Berlin, 1984).

[12] D. S. Dean, J. Phys. A 29, L613 (1996).

[13] U. M. B. Marconi and P. Tarazona, J. Chem. Phys. 110, 8032 (1999); A. J. Archer and M. Rauscher, J. Phys. A 37, 9325 (2004).

[14] M. A. Fuentes, M. N. Kuperman, and V. M. Kenkre, Phys. Rev. Lett. 91, 158104 (2003); E. Hernández-García and C. López, Phys. Rev. E 70, 016216 (2004); C. López and E. Hernández-García, Physica D 199, 223 (2004); M. G. Clerc, D. Escaff, and V. M. Kenkre, Phys. Rev. E 72, 056217 (2005).

[15] According to the kinetic interpretation, the discrete-time version of the Langevin equation Eq. (10) goes through postpoint discretization. However, in order to have a dynamics of nonoverlapping generations we write down first its equivalent Langevin equation in the Ito formulation, Eq. (11), and then consider its time-discrete form to order $\Delta t$. 